\title{
Inhibition of NF- $\kappa$ B activation and MMP-9 secretion by plasma of human volunteers after ingestion of maritime pine bark extract (Pycnogenol)
}

\author{
Tanja Grimm¹, Zuzana Chovanová2, Jana Muchová2, Katarína Sumegová2, \\ Anna Liptáková2 ${ }^{2}$ Zdeňka Ďuračková ${ }^{2}$ and Petra Högger*1
}

\begin{abstract}
Address: ${ }^{1}$ Institut für Pharmazie und Lebensmittelchemie, Bayerische Julius-Maximilians-Universität, Würzburg, Germany and ${ }^{2}$ Department of Medical Chemistry, Biochemistry and Clinical Biochemistry, Faculty of Medicine, Comenius University, Bratislava, Slovak Republic

Email: Tanja Grimm - hogger@pzlc.uni-wuerzburg.de; Zuzana Chovanová - zuzana.chovanova@fmed.uniba.sk; Jana Muchová - zdenka.durackova@fmed.uniba.sk; Katarína Sumegová - zdenka.durackova@fmed.uniba.sk; Anna Liptáková - zdenka.durackova@fmed.uniba.sk; Zdeňka Ďuračková - zdenka.durackova@fmed.uniba.sk; Petra Högger* - hogger@pzlc.uniwuerzburg.de

* Corresponding author
\end{abstract}

Published: 27 January 2006

Received: 05 November 2005

Journal of Inflammation2006, 3:1 doi:10.1186/1476-9255-3-I

Accepted: 27 January 2006

This article is available from: http://www.journal-inflammation.com/content/3/I/I

(C) 2006Grimm et al; licensee BioMed Central Ltd.

This is an Open Access article distributed under the terms of the Creative Commons Attribution License (http://creativecommons.org/licenses/by/2.0), which permits unrestricted use, distribution, and reproduction in any medium, provided the original work is properly cited.

\begin{abstract}
French maritime pine bark extract (Pycnogeno ${ }^{\circledR}$ ) displays a variety of anti-inflammatory effects in vivo. Aim of this study was to determine whether human plasma after oral intake of Pycnogenol contains sufficient concentrations of active principles to inhibit key mediators of inflammation. Blood samples from seven healthy volunteers were obtained before and after five days administration of $200 \mathrm{mg}$ Pycnogenol per day. Plasma samples statistically significantly inhibited matrix metalloproteinase 9 (MMP-9) release from human monocytes and NF- $\mathrm{KB}$ activation. Thus, we provide evidence that bioavailable active principles of Pycnogenol exert anti-inflammatory effects by inhibition of proinflammatory gene expression which is consistent with documented clinical observations. We suggest that our ex vivo method is suitable to substantiate molecular pharmacological mechanisms of complex plant extracts in a more focussed and rational way compared to in vitro studies by taking into account the processes of absorption and metabolism.
\end{abstract}

\section{Background}

Pycnogenol is a standardized bark extract of the French maritime pine Pinus pinaster (Pycnogenol ${ }^{\oplus}$, Horphag Research Ltd., UK). It comprises of a concentrate of pine bark constituents such as polyphenolic monomers, procyanidins and phenolic or cinnamic acids and their glycosides [1]. About $65-75 \%$ of the Pycnogenol extract are procyanidins that consist of catechin and epicatechin subunits of varying chain lengths [1]. The quality of this extract is specified in the United States Pharmacopeia (USP 28) [2].
In human studies Pycnogenol revealed diverse antiinflammatory actions [1]. Double-blind, placebo-controlled studies in asthma patients showed reduced plasma [3] or urine [4] leukotriene concentrations after Pycnogenol supplementation, while asthma symptom scores and pulmonary function improved. Symptoms of osteoarthritis as pain and immobility of joints decreased in a doubleblind, placebo-controlled study [5]. Oral [6] and topical [7] application of Pycnogenol reduced inflammation and delayed skin-cancer formation following UV-radiation in controlled experiments in mice. 

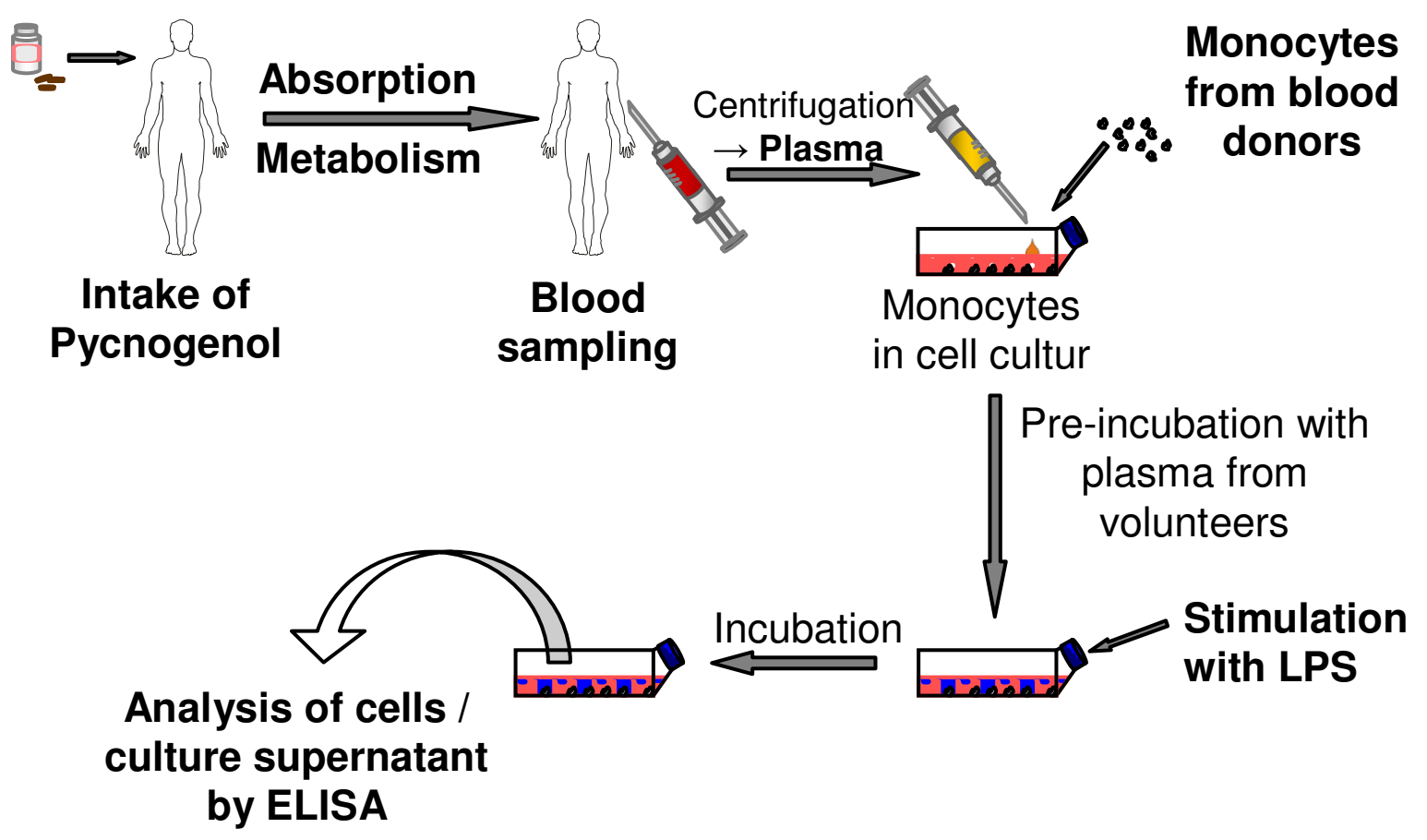

Figure I

Schematic representation of the experimental procedure of the ex vivo experiments with human plasma incubated with monocytes.

The anti-inflammatory mechanisms of maritime pine bark extract have been elucidated in a variety of in vitro and cell culture studies [8,9]. Additionally to its radical scavenging activity an inhibition of NF-kB-dependent gene expression and decrease of the activity of various pro-inflammatory mediators and adhesion molecules was observed after incubation of cells with the Pycnogenol extract $[8,9]$. This experimental in vitro design that pursues to uncover pharmacological effects by addition of plant extracts to cell cultures and subsequent measurement of cellular responses is widely employed. However, this methodology might inherit a couple of pitfalls.

Plant extracts often comprise of high molecular weight components that cannot be absorbed in the gastrointestinal tract and thus will never reach a target cell in vivo. Furthermore, there are examples of metabolites that are not present in the original extract, but are formed in vivo as a result of intestinal bacterial and/or hepatic metabolism. After ingestion of Pycnogenol, for example, two metabolites derived from catechin were detected in human urine, $\delta$-(3,4-dihydroxy-phenyl)- $\gamma$-valerolactone and $\delta$-(3-methoxy-4-hydroxy-phenyl)- $\gamma$-valerolactone [10]. Valerolactone derivatives were also found after ingestion of green tea [11]. These newly formed metabolites may display significant efficacy and contribute to the observed in vivo effects. We recently elucidated the cellular effects of $\delta$ (3,4-dihydroxy-phenyl)- $\gamma$-valerolactone and $\delta$-(3-methoxy-4-hydroxy-phenyl)- $\gamma$-valerolactone and uncovered an antioxidant activity as well as the potential to inhibit release and enzymatic activity of matrix metalloproteinase 9 (MMP-9) [12].

Thus, pharmacokinetic issues of absorption and metabolism should be considered for valid identification of molecular pharmacological effects of plant extracts. A methodological approach that considers both the absorption and possible metabolism of plant extract components would involve laboratory animals or human volunteers who donate blood samples. These blood samples should contain all bioavailable active principles of the extract and allow an ex vivo analysis in all kind of molecular pharmacological effects in cell culture assays (Figure 1). There are only few examples of experimental settings described in literature that use this approach. Effects of nettle herb [13] or willow bark extract [14] on cytokine release and effect of Harpagophytum extract on eicosanoid biosynthesis [15] were elucidated in whole- 
blood assays of human volunteers after ingestion of the extract. Recently, a potent ex vivo anti-HIV activity was detected in sera of volunteers after administration of Phyllanthus amarus plant material [16].

The purpose of the present study was to determine molecular pharmacological effects of maritime pine bark extract ex vivo after intake of regular doses by human volunteers. Therefore, we obtained plasma samples before and after five days administration of Pycnogenol to seven healthy humans. These plasma samples were analyzed in two different experimental settings to evaluate the influence of bioavailable actives principles on cellular key components that contribute to inflammatory processes. We investigated a potential influence of the plasma samples on LPS-induced release of MMP-9 from human monocytes. Since MMP-9 induction and release might be initiated by NF- $\mathrm{B}$ activation we also determined the effect of the plasma samples on LPS-induced NF- $\mathrm{kB}$ nuclear translocation.

\section{Methods \\ Patients}

Seven healthy volunteers (five female and two male) aged 18 to 30 years participated in this study. The study was approved by the ethical committee of the Comenius University's Faculty of Medicine, Bratislava, Slovak Republic, and all participants gave written informed consent. After 24 hours of a diet free of flavonoids (no vegetables, fruits and fruit juices or marmalades, tea, coffee, cocoa, wine and beer) blood samples were drawn to obtain basal values. Subsequently, the volunteers took tablets containing $200 \mathrm{mg}$ standardized maritime pine bark extract (Pycnogenol ${ }^{\circledR}$, Horphag Research Ltd., UK) every morning for five days to reach steady state conditions of constituents and/ or metabolites of Pycnogenol. Four hours after the last intake of Pycnogenol on day five a second blood sample was obtained from each volunteer. Again, a 24 hour period of a diet free of flavonoids preceded this blood sampling. Blood samples were centrifuged and plasma was aliquoted, shock frozen and stored at $-80^{\circ} \mathrm{C}$ until further analysis.

\section{Isolation and culture of human monocytes}

Human monocytes were isolated from pooled blood cell suspensions (Bayerisches Rotes Kreuz, Wiesentheid, Germany) from different donors by Biocoll (Biochrom, Berlin, Germany) and subsequent Percoll (Pharmacia, Freiburg, Germany) density gradient centrifugation. Only blood cell suspensions of donors with blood type 0 were used for these experiments. The cells were first cultured overnight in Mc Coy's 5a modified medium (Biochrom, Berlin, Germany) supplemented with $15 \%$ fetal calf plasma, $1 \%$ penicilline/streptomycine, $1 \%$ non-essential amino acids and $1 \mathrm{mM}$ L-glutamine at a density of $5 \times 10^{6}$
cells/mL (NF-kB experiments) or $1 \times 10^{6}$ cells $/ \mathrm{mL}$ (MMP9 experiments) in a $6 \% \mathrm{CO}_{2}$ humidified atmosphere at $37^{\circ} \mathrm{C}$ (Hera cell incubator, Kendro Laboratory Products, Hanau, Germany). Cell experiments were performed in Multiwell $^{\mathrm{TM}}$ 24-well cell culture plates, polystyrene, (BD Labware NJ, USA) with a final volume of $2.0 \mathrm{~mL} /$ well.

\section{Inhibition of MMP-9 release from human monocytes}

Plasma samples obtained before and after Pycnogenol intake were diluted 1:1 with RPMI medium (Biochrom, Berlin, Germany; supplemented with $1 \%$ penicilline/ streptomycine, $1 \%$ non-essential amino acids and $1 \mathrm{mM}$ L-glutamine) and incubated with monocytes for one hour. Cells were then stimulated with $10 \mathrm{ng} / \mathrm{ml}$ LPS (Lipopolysaccharides (rough strains) from Salmonella minnesota Re 595, Sigma-Aldrich Inc., Taufkirchen, Germany) and incubated at $37^{\circ} \mathrm{C}$ for 48 hours. The number of viable cells was determined by counting living cells after staining with trypane blue. Plates were centrifuged (Megafuge 1.0 R, Kendro Laboratory Products) and cell culture supernatants were harvested, diluted 1:25 and assayed for total MMP-9 protein concentrations by ELISA (Quantikine ${ }^{\mathrm{TM}}$ assay, R\&D Systems, Minneapolis, USA) according to manufacturer's protocol.

\section{Determination of NF- $\kappa$ B activation by ELISA}

Plasma samples obtained before and after Pycnogenol intake were diluted 1:1 with RPMI medium as described above and incubated with monocytes overnight. Cells were then stimulated with $1 \mu \mathrm{g} / \mathrm{mL}$ LPS and incubated at $37^{\circ} \mathrm{C}$ for 60 minutes. After incubation the number of viable cells was determined by counting living cells after staining with trypane blue. Determination of free p65 in nuclear extracts was performed according to the manufacturer's protocol (ELISA-Kit NF- $\kappa$ B p65 ActivELISA ${ }^{\mathrm{Tm}}$, Imgenex, CA, USA). The optical density of samples was determined using the microplate reader (Bio-Rad microplate reader, Benchmark CA, USA) set at $405 \mathrm{~nm}$. Inhibitory effects of plasma constituents and metabolites after Pycnogenol intake were determined by comparing the p65 concentration of LPS-stimulated cells, incubated with plasma before and after Pycnogenol intake.

\section{Statistical analysis}

Statistical analysis (Wilcoxon matched pairs signed rank test) was performed using the GraphPad prism software (GraphPad Software Inc., San Diego CA, USA). Significance was defined as $\mathrm{p}<0.05$.

\section{Results}

Human monocytes were incubated with diluted plasma samples (dilution 1:1 with cell culture medium) obtained from seven healthy volunteers before and after ingestion of maritime pine bark extract (Figure 1). The viability of monocytes was not significantly influenced by plasma 

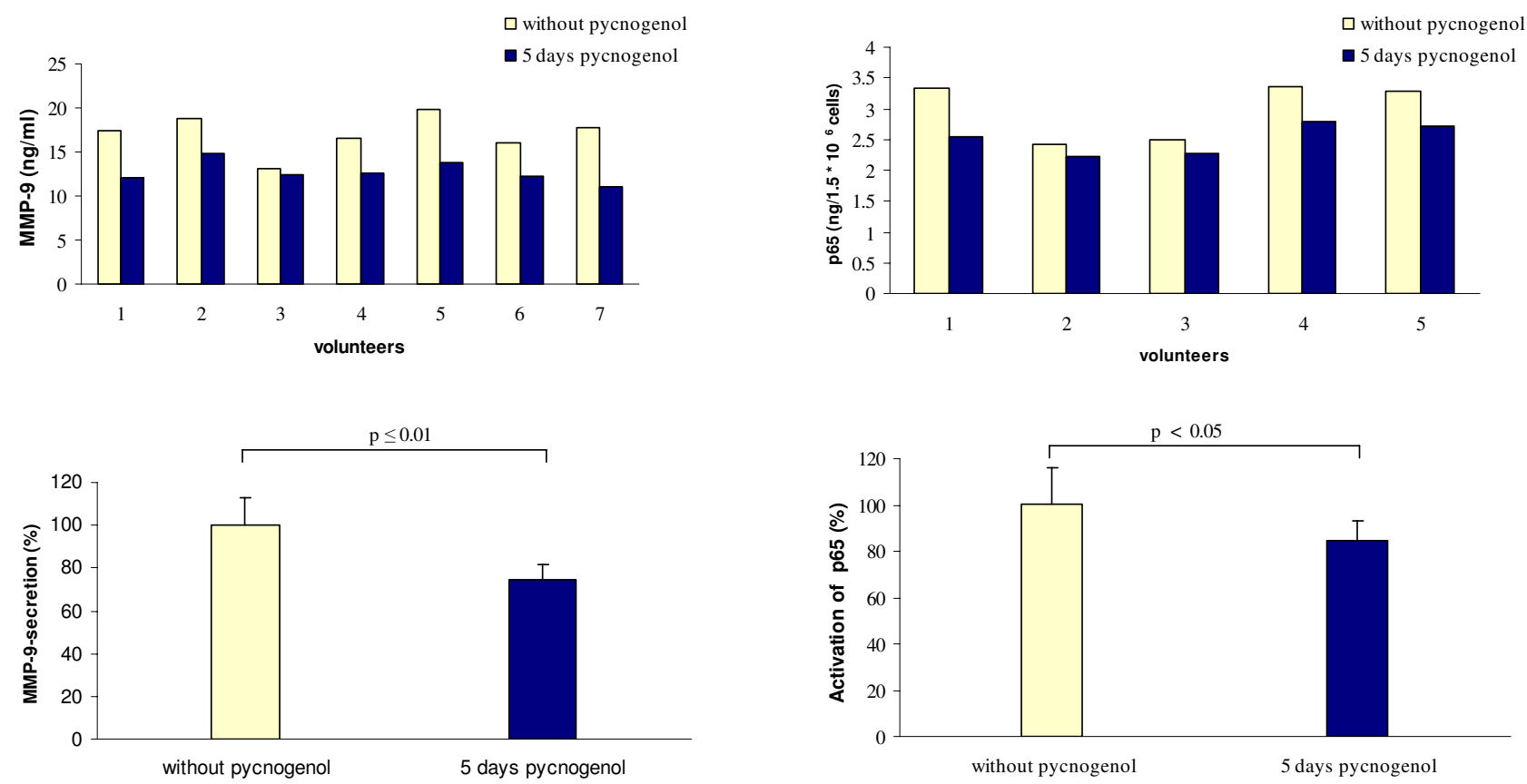

\section{Figure 2}

Inhibition of LPS-induced matrix metalloproteinase 9 (MMP9) from human monocytes by plasma of seven volunteers before and after five days intake of $200 \mathrm{mg}$ maritime pine bark extract (Pycnogenol). The upper panel shows concentrations of MMP-9 in cell culture supernatants of $2.5 \times 10^{5}$ viable cells after ex vivo incubation with the individual volunteers' plasma. The lower panel displays mean and standard deviation of percentage MMP-9 release. It statistically significantly reduced by plasma samples after administration of Pycnogenol ( $p<0.01$, Wilcoxon matched pairs signed rank test).

samples obtained from Pycnogenol treated subjects. In the MMP-9 experiments the number of viable monocytes was $2.49 \pm 0.23 \times 10^{5}$ after incubation with samples obtained before Pycnogenol intake and $2.79 \pm 0.26 \times 10^{5}$ after incubation with plasma obtained after 5 days Pycnogenol ingestion. Likewise, no difference was observed in the number of viable cells in the NF- $\kappa \mathrm{B}$ experiments. The number of viable monocytes was $1.49 \pm 0.29 \times 10^{6}$ and $1.70 \pm 0.20 \times 10^{6}$ after incubation with samples obtained before and after 5 days Pycnogenol intake.

A statistically significant decrease of MMP-9 concentration in cell culture supernatant was induced by the plasma samples obtained after intake of Pycnogenol compared to basal values (Figure 2). The mean MMP-9 concentration after LPS challenge of monocytes incubated with volunteers' plasma samples obtained before Pycnogenol ingestion was $17.06 \pm 2.17 \mathrm{ng} / \mathrm{mL}$ per $2.5 \times 10^{5}$ viable human monocytes. This concentration was reduced to $12.70 \pm$

\section{Figure 3}

Inhibition of LPS-induced NF- $\kappa B$ activation by plasma of five volunteers before and after five days intake of $200 \mathrm{mg}$ maritime pine bark extract (Pycnogenol). The upper panel shows concentrations of p65 was determined in nuclear extracts of $1.5 \times 10^{6}$ viable human monocytes after ex vivo incubation with the individual volunteers' plasma. The lower panel displays mean and standard deviation of percentage nuclear concentration of $p 65$. It was statistically significantly reduced by plasma samples after administration of Pycnogenol $(p<$ 0.05, Wilcoxon matched pairs signed rank test).

$1.24 \mathrm{ng} / \mathrm{mL}$ when monocytes were incubated with plasma obtained after 5 days Pycnogenol intake. This corresponded to a mean decrease in MMP-9 concentration of $25 \%$. The plasma of all study participants exhibited an inhibitory effect on LPS-induced MMP-9 secretion, but interindividual variations were obvious. The inhibitory effect ranged from $4.6 \%$ to $39 \%$ inhibition.

A statistically significant reduction of nuclear p65 concentration was observed when human monocytes were exposed to plasma (dilution 1:1 with cell culture medium) obtained after intake of Pycnogenol compared to basal values (Figure 3). For this experiment, sufficient volumes of plasma were only available from five volunteers; two plasma samples had been used up for repetition experiments after cell culture contamination. The mean nuclear p65 concentration after LPS challenge was $2.98 \pm$ $0.48 \mathrm{ng}$ per $1.5 \times 10^{6}$ viable human monocytes. This nuclear concentration was reduced to $2.51 \pm 0.26 \mathrm{ng}$ per 


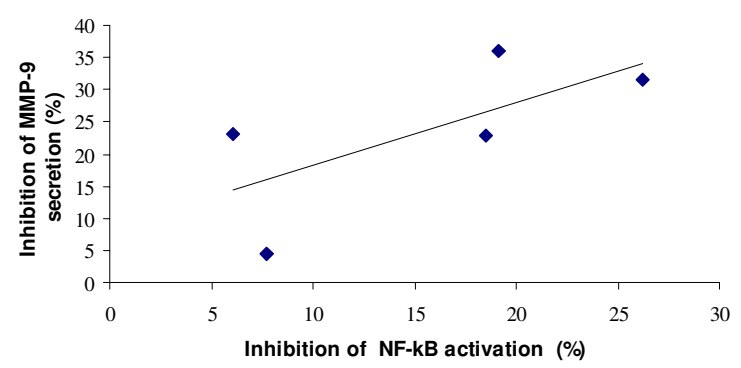

\section{Figure 4}

Correlation of inhibition of LPS-induced NF- $\kappa B$ activation and MMP-9 release by matching plasma samples of five volunteers before and after five days intake. Coefficient of correlation was 0.6 (Spearman rank correlation coefficient).

$1.5 \times 10^{6}$ viable cells when monocytes were incubated with plasma obtained after 5 days Pycnogenol intake. The plasma of all study five participants exhibited an inhibitory effect on LPS-induced NF- $\kappa \mathrm{B}$ activation, but interindividual variations were obvious. The inhibitory effect ranged from $6 \%$ to $25 \%$ with a mean of $15.5 \%$ inhibition.

For five volunteers whose plasma samples were used for both the MMP-9 secretion and NF- $\kappa \mathrm{B}$ experiments a correlation of their plasmas' inhibitory activity on MMP-9 secretion and NF- $\kappa \mathrm{B}$ nuclear translocation was calculated (Figure 4). The correlation (Spearman rank correlation coefficient) was positive $(r=0.6)$ though not statistically significant due to limited number of samples.

\section{Discussion}

Plant extracts may display a variety of interesting pharmacological effects in vivo. The bioefficacy of plant extracts is increasingly tested and documented in clinical intervention studies [17]. While the efficacy of extracts is observed with increasing interest the elucidation of the molecular basis of biological or clinical effects remains a challenge. Usually plant extracts comprise of a complex mixture of various components and often enough it is not clear whether a single compound or a mixture of related compounds is responsible for the effects.

The standardized maritime pine bark extract Pycnogenol has documented clinical anti-inflammatory activities $[1,9]$. In earlier studies we determined that the extract's metabolites $\delta$-(3,4-dihydroxy-phenyl)- $\gamma$-valerolactone and $\delta$-(3-methoxy-4-hydroxy-phenyl)- $\gamma$-valerolactone exhibited inhibitory activity on LPS-induced secretion of matrix metalloproteinase 9 (MMP-9) from human monocytes [12]. However, so far it remained elusive whether sufficiently high in vivo concentrations of any active extract components would be achieved after peroral intake of Pycnogenol. In the present study we applied an ex vivo methodology that takes absorption and metabolism of the extract into account. The plasma samples obtained from volunteers after ingestion of Pycnogenol were expected to contain active extract components that should attenuate inflammatory processes.

Indeed, we observed a statistically significant mean decrease of about $25 \%$ in MMP-9 release when LPS-activated human monocytes were exposed to plasma of volunteers after repeated intake of Pycnogenol. The matrix degrading enzyme MMP-9 is highly expressed at sites of inflammation and contributes to the pathogenesis of various chronic inflammatory diseases. In asthma MMP-9 is upregulated and involved in remodeling processes [1820]. MMP-9 also facilitates recruitment of inflammatory cells such as eosinophils and neutrophils across basement membranes [18]. Expression of MMP-9 was negatively correlated with pulmonary function in asthmatic patients [20]. As Pycnogenol has been reported to attenuate signs of inflammation in asthma patients $[3,4]$ we now provide first evidence that this anti-inflammatory in vivo effect might be at least partially attributed to reduced MMP-9 secretion on a molecular level.

$\mathrm{NF}-\kappa \mathrm{B}$ is a molecule with a master function in inflammatory cytokine induction. It is also involved in regulation of immune functions, cell cycle control and apoptosis [21]. Upon nuclear translocation in response to an inflammatory stimulus it regulates various genes coding for proinflammatory mediators. We found that plasma of Pycnogenol treated volunteers statistically significantly inhibited NF- $\kappa \mathrm{B}$ activation in LPS-stimulated monocytes by about $15 \%$. Though this effect is rather moderate it might well contribute to the anti-inflammatory effects of Pycnogenol in patients. Interestingly, $\mathrm{NF}-\kappa \mathrm{B}$ is also involved in MMP-9 expression $[22,23]$. Consistent with these reports we observed a positive correlation between inhibitory activity of matched plasma samples on MMP-9

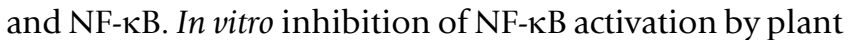
extracts or constituents has been reported repeatedly [24]. Blocking $\mathrm{I} \kappa \mathrm{B}$ kinase activity has been reported as the underlying mechanism for restricting NF- $\kappa \mathrm{B}$ activation by green tea polyphenols [25]. The mechanism of ex vivo inhibition of NF- $\mathrm{B}$ nuclear translocation by plasma containing bioavailable active principles after Pycnogenol ingestion has yet to be identified.

To summarize, regular doses of perorally administered French maritime pine bark extract moderately inhibited NF- $\kappa \mathrm{B}$ activation and MMP-9 secretion ex vivo. Since the plasma samples of the volunteers were diluted 1:1 with cell culture medium before incubation with the mono- 
cytes it can be assumed that in vivo effects might be even more pronounced. The observed ex vivo effects with plasma of volunteers after Pycnogenol intake are consistent with reported clinical anti-inflammatory effects in vivo. The next challenge will be to identify the responsible active principle(s) in the plasma samples. After all, however, the suggested methodology is a rational and focussed technique to explain biological effects from in vivo studies on a molecular pharmacological basis. The next target will be to link pharmacodynamic data with pharmacokinetics and to identify the active component(s) in plasma samples.

\section{Competing interests}

This work was supported by a research grant of Horphag Research. Z.D. was additionally supported by a VEGA grant of the Ministry of Education of Slovak Republic and by Mind \& Health civil association.

\section{Authors' contributions}

T.G. carried out all experiments with the plasma samples and the data analysis.

Z.C. recruited the volunteers and organized the study, prepared the technical documentation for blood sampling.

J.M. and K.S. took care of the volunteers and performed blood sampling and processed samples according to the protocol.

A.L. prepared the project and processed blood samples.

Z.D. contributed to planning of the design and execution of the project and wrote the ethic's committee application.

P.H. conceived of and designed the study and wrote the manuscript.

All authors read and approved the final manuscript.

\section{References}

I. Rohdewald P: A review of the French maritime pine bark extract (Pycnogenol), a herbal medication with a diverse clinical pharmacology. Int ] Clin Pharmacol Ther 2002, 40: I58-I68.

2. Maritime Pine Extract. In United States Pharmacopeia Volume 28. Rockville, United States Pharmacopeial Convention, Inc.; 2005:2115-2116.

3. Hosseini S, Pishnamazi S, Sadrzadeh SM, Farid F, Farid R, Watson RR: Pycnogenol((R)) in the Management of Asthma. J Med Food 200I, 4:20I-209.

4. Lau BH, Riesen SK, Truong KP, Lau EW, Rohdewald P, Barreta RA: Pycnogenol as an adjunct in the management of childhood asthma. J Asthma 2004, 41:825-832.

5. Farid R, Mirteizi A, Mirheidari M, Rezaieyazdi S, Mansouritorghabeh $\mathrm{H}$, Zibadi S, Watson RR: Pycnogenol $囚$ reduces clinical symptoms of knee osteoarthritis. Arthritis Care Res . accepted for publication

6. Kyriazi M, Yova D, Rallis M, Lima A: Cancer chemopreventive effects of Pinus Maritima bark extract on ultraviolet radia- tion and ultraviolet radiation-7, I2, dimethylbenz(a)anthracene induced skin carcinogenesis of hairless mice. Cancer Lett 2005.

7. Sime S, Reeve VE: Protection from inflammation, immunosuppression and carcinogenesis induced by UV radiation in mice by topical Pycnogenol. Photochem Photobiol 2004, 79:193-198.

8. Packer L, Rimbach G, Virgili F: Antioxidant activity and biologic properties of a procyanidin-rich extract from pine (Pinus maritima) bark, pycnogenol. Free Radic Biol Med 1999, 27:704-724.

9. Rohdewald PJ: Pycnogenol, French Maritime Pine Bark Extract. In Encyclopedia of Dietary Supplements New York, Marcel Dekker; 2005:545-553.

10. Düweler KG, Rohdewald P: Urinary metabolites of French maritime pine bark extract in humans. Pharmazie 2000, 55:364-368.

II. Meng X, Sang S, Zhu N, Lu H, Sheng S, Lee MJ, Ho CT, Yang CS: Identification and characterization of methylated and ring-fission metabolites of tea catechins formed in humans, mice, and rats. Chem Res Toxicol 2002, I 5:1042-1050.

12. Grimm T, Schäfer A, Högger P: Antioxidant activity and inhibition of matrix metalloproteinases by metabolites of maritime pine bark extract (pycnogenol). Free Radic Biol Med 2004, 36:8II-822.

13. Teucher T, Obertreis B, Ruttkowski T, Schmitz H: [Cytokine secretion in whole blood of healthy subjects following oral administration of Urtica dioica L. plant extract]. Arzneimittelforschung 1996, 46:906-910.

14. Wagner I, Greim C, Laufer S, Heide L, Gleiter $\mathrm{CH}$ : Influence of willow bark extract on cyclooxygenase activity and on tumor necrosis factor alpha or interleukin I beta release in vitro and ex vivo. Clin Pharmacol Ther 2003, 73:272-274.

15. Loew D, Mollerfeld J, Schrodter A, Puttkammer S, Kaszkin M: Investigations on the pharmacokinetic properties of Harpagophytum extracts and their effects on eicosanoid biosynthesis in vitro and ex vivo. Clin Pharmacol Ther 200I, 69:356-364.

16. Notka F, Meier G, Wagner R: Concerted inhibitory activities of Phyllanthus amarus on HIV replication in vitro and ex vivo. Antiviral Res 2004, 64:93-102.

17. Williamson G, Manach C: Bioavailability and bioefficacy of polyphenols in humans. II. Review of $\mathbf{9 3}$ intervention studies. Am J Clin Nutr 2005, 8 I :243S-255S.

18. Lee YC, Lee HB, Rhee YK, Song $\mathrm{CH}$ : The involvement of matrix metalloproteinase-9 in airway inflammation of patients with acute asthma. Clin Exp Allergy 200I, 31:1623-1630.

19. Kelly EA, Jarjour NN: Role of matrix metalloproteinases in asthma. Curr Opin Pulm Med 2003, 9:28-33.

20. Wenzel SE, Balzar S, Cundall M, Chu HW: Subepithelial basement membrane immunoreactivity for matrix metalloproteinase 9: association with asthma severity, neutrophilic inflammation, and wound repair. J Allergy Clin Immunol 2003, I II:I345-1352.

21. Celec P: Nuclear factor kappa B--molecular biomedicine: the next generation. Biomed Pharmacother 2004, 58:365-37I.

22. Chung TW, Moon SK, Chang YC, Ko JH, Lee YC, Cho G, Kim SH, Kim JG, Kim CH: Novel and therapeutic effect of caffeic acid and caffeic acid phenyl ester on hepatocarcinoma cells: complete regression of hepatoma growth and metastasis by dual mechanism. Faseb J 2004, 18:1670-168I.

23. Ho TY, Bagnell CA: Relaxin Induces Matrix Metalloproteinase9 through Activation of Nuclear Factor Kappa B in Human THP-I Cells. Ann N Y Acad Sci 2005, 1041:314-316.

24. Kim HP, Son KH, Chang HW, Kang SS: Anti-inflammatory plant flavonoids and cellular action mechanisms. J Pharmacol Sci 2004, 96:229-245.

25. D'Acquisto F, May MJ, Ghosh S: Inhibition of Nuclear Factor Kappa B (NF-B): An Emerging Theme in Anti-Inflammatory Therapies. Mol Interv 2002, 2:22-35. 